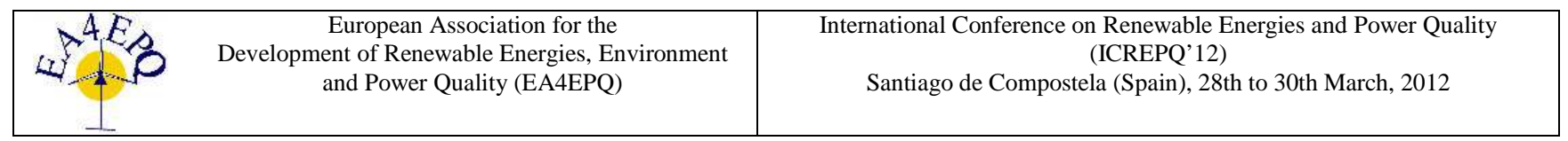

\title{
2D Vibration based MEMS Energy Harvester
}

\author{
Sehwan Kim $^{1}$ and Kukjin Chun ${ }^{1}$ \\ ${ }^{1}$ Department of Electrical Engineering and Computer Science \\ Seoul National University \\ ${ }^{1}$ Inter-university Semiconductor Research Center \\ San 56-1, Shinlim-dong, Kwanak-gu, Seoul 151-742, Korea \\ Phone number: +82 2880 1811, e-mail: ksh@mintlab.snu.ac.kr, kchun@snu.ac.kr
}

\begin{abstract}
Electrostatic energy harvester for two axis vibration is developed. It was fabricated by using silicon-glass anodic bonding and deep silicon etching. The device which generates energy from only one directional vibration has much different resonant frequency for each direction. To gain energy from two or more directional vibration the structure was designed to have equal resonant frequency along the $\mathrm{x}$, $\mathrm{y}$-axis by symmetric spring-mass structure. When the direction of ambient vibration is changed from $\mathrm{x}$-axis to $\mathrm{y}$-axis, the device still generates equal amount of energy. Resonant frequency of the structure is $191 \mathrm{~Hz}$ along the $\mathrm{x}$-axis vibration direction. The device size is $9.0 \mathrm{~mm} \times 9.0 \mathrm{~mm} \times 0.6 \mathrm{~mm}$. Vibration of which the acceleration is $1.2 \mathrm{~m} / \mathrm{s}^{2}$ and the frequency is $191 \mathrm{~Hz}$ generates $0.73 \mu \mathrm{W} / \mathrm{cm}^{3}$. Although vibration direction is not matched, power is generated more than $0.2 \mu \mathrm{W} / \mathrm{cm}^{3}$ at resonant frequency.
\end{abstract}

\section{Key words}

Energy Harvester, two-axis, vibration

\section{Introduction}

Batteries are widely used as energy sources because they have small size, portability, stability. However, their lifetime is not permanent. They should be recharged or replaced periodically. Energy harvester is one of the alternatives. It generates energy from sunlight, vibration, heat and etc. It has semi-permanent lifetime and doesn't need to recharge or replace.[1-2]

Vibration-based energy harvester is the device which generates electrical energy from vibration energy. It has electrostatic, electromagnetic, or piezoelectric transducer. Each type uses change of capacitance, electromagnetic induction[3-4], and piezoelectric characteristics by vibration acceleration[5-6]. Although electrostatic type needs voltage source, but it is easily integrated with microsystems[7].

Many researches about vibration-based energy harvesters are conducted. In many cases they use mechanical resonation of mass-spring structure. To generate maximum power from energy harvester, matching direction and frequency of vibration are needed. However, in many cases direction of the vibration is unknown. Also it can be changed temporarily. If the direction of the vibration and the device are not matched, efficiency is considerably decreased. We designed electrostatic energy harvester for two-axis vibration. It can generate energy although the direction of the vibration is changed from $\mathrm{x}$ axis to y-axis.

\section{Design}

To generate energy from two-axis vibration mass-spring structure has same resonant frequency along $\mathrm{x}$-axis and $y$-axis. So the structure of the device should be designed symmetrically. Fig. 1 is a schematic of the energy harvester for two-axis vibration. One of the spring directions is $\mathrm{x}$-axis and the other is y-axis. It was designed to have same frequency along the $\mathrm{x}$-axis and $\mathrm{y}$ axis. Four fixed electrodes are electrically connected and make capacitance with mass. Stopper limits displacement of the mass and avoids contacts between comb fingers. ANSYS simulation is used to find resonant frequency of the structure $200 \mathrm{~Hz}$. Four springs connects anchors and a mass. When the acceleration is applied to the device, each comb fingers get closer and capacitance is changed.

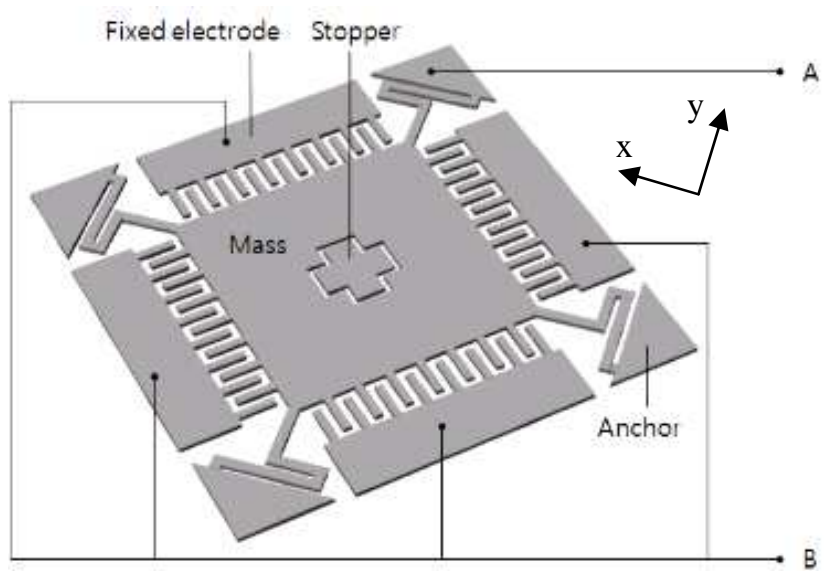

Fig. 1. Schematic of electrostatic energy harvester for two-axis vibration 


\section{Fabrication}

The electrostatic energy harvester is fabricated by bulk micromachining. Fig. 2 shows fabrication process flow. First, PECVD TEOS oxide is patterned on the silicon wafer. Using TEOS oxide as hard mask, silicon is etched by deep reactive ion etching(DRIE). TEOS oxide is stripped and silicon-glass anodic bonding is conducted. TEOS oxide and Ti/Au is patterned on the silicon. Ti/Au layer is used as electrodes. Finally silicon is etched by DRIE and the structure is released. Because it doesn't use wet release process, no stiction problem is occured. Fig. 3 shows photograph of the fabricated energy harvester structure. Size of the device is $9.0 \mathrm{~mm} \times 9.0 \mathrm{~mm} \times 0.6 \mathrm{~mm}$.

\section{Silicon wafer}

1. TEOS oxide patterning

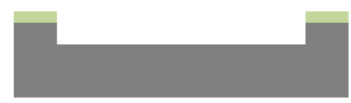

2. Deep silicon etch

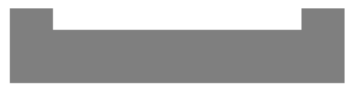

3. TEOS oxide strip

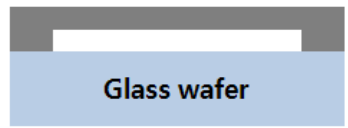

4. Anodic bonding, Si CMP

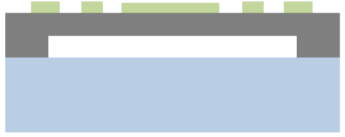

5. TEOS oxide patterning

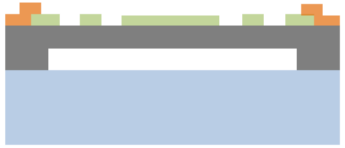

6. Ti/Au patterning (lift off)

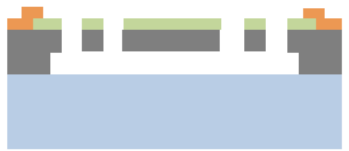

7. Deep silicon etch
Fig. 2. Fabrication process of the electrostatic energy harvester

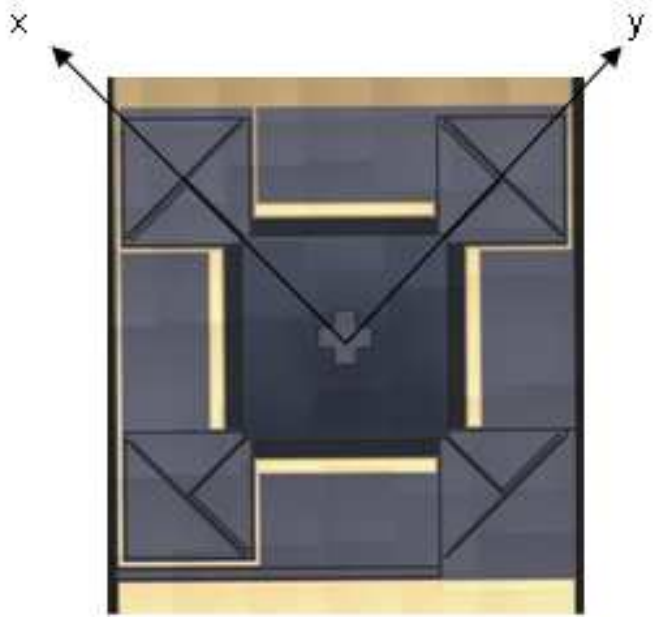

Fig. 3. Photograph of fabricated energy harvester structure

\section{Measurement Result}

Measurement setting consists of a vibrator, an accelerometer and measurement circuit. Vibrator makes vibration with particular frequency. Accelerometer measures acceleration and frequency of the sample. To supply charge to energy harvester device, voltage source

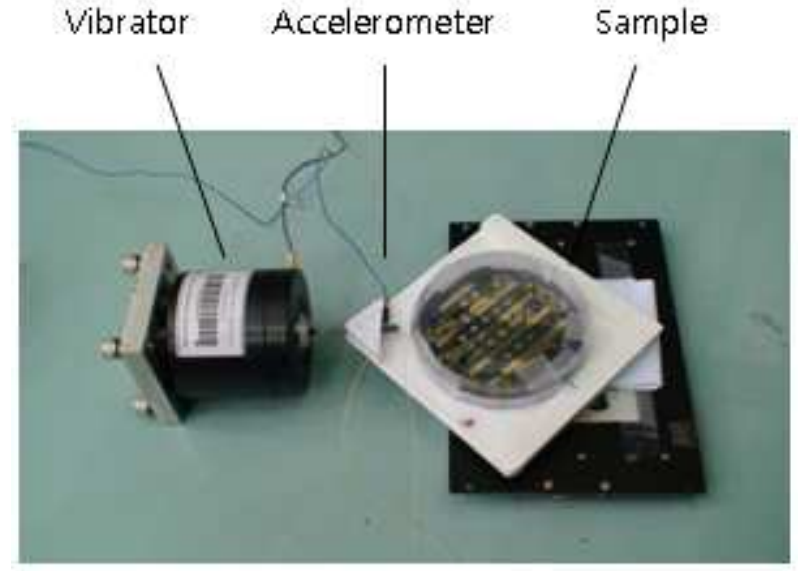

Fig. 4. Measurement setup for the energy harvester

was used. Fig. 4 shows measurement setup for the fabricated energy harvester. The energy harvester device sample is excited laterally. The sample is connected to output circuit.

Resonant frequency of the device at each direction of the vibration is measured. The direction of the vibration is expressed as an clockwise angle from $\mathrm{x}$-axis. Resonant frequency is $191 \mathrm{~Hz}$ at $0,90^{\circ}, 194 \mathrm{~Hz}$ at $45,135^{\circ}$, and $197 \mathrm{~Hz}$ at $22.5,67.5,112.5,157.7^{\circ}$. At angle $0^{\circ}$, power densities are measured for each vibration frequency. Source voltage is $4.48 \mathrm{~V}$ and excited vibration acceleration is $1.2 \mathrm{~m} / \mathrm{s}^{2}$. Fig. 5 shows a graph of power densities versus vibration frequency. At resonant frequency $191 \mathrm{~Hz}$, the device generates $35 \mathrm{nW}$ and power density is $0.73 \mu \mathrm{W} / \mathrm{cm}^{3}$. Output voltage ripple is $34 \mathrm{mV}$ and average output voltage is $5.04 \mathrm{~V}$. Fig. 6 shows the power measurement result at each acceleration. As acceleration of vibration is increased, generated power is also increased. For $191 \mathrm{~Hz}$ resonant frequency, acceleration $1.2 \mathrm{~m} / \mathrm{s}^{2}$ makes maximum power density for the device. Over $1.2 \mathrm{~m} / \mathrm{s}^{2}$ acceleration, the measured power density is varied for each experiment because a stopper at the center of the device disturbs the movement of the mass. Fig. 7 shows the result of power density versus angle between $\mathrm{x}$-axis and vibration direction. Although vibration direction is not matched, power is generated more than $0.2 \mu \mathrm{W} / \mathrm{cm}^{3}$ at resonant frequency.

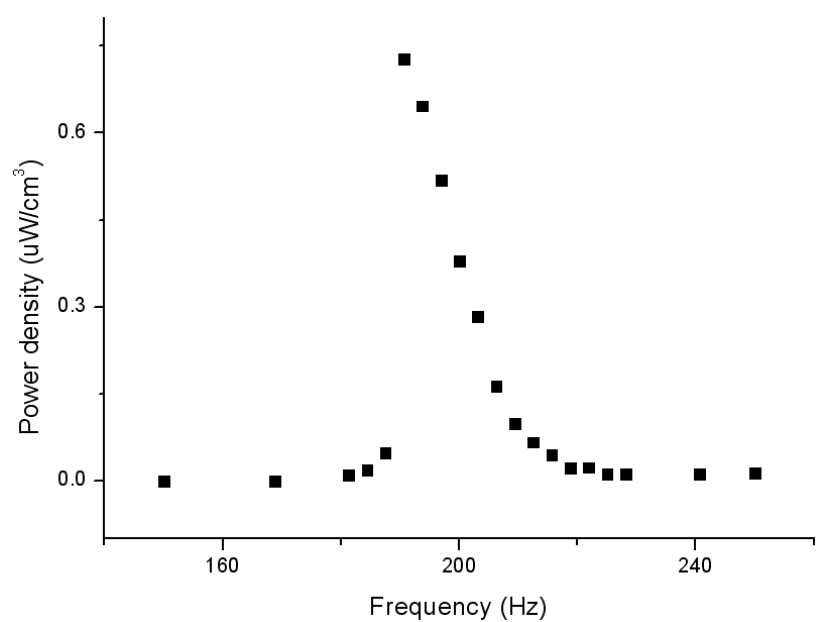

Fig. 5. Graph of power density versus vibration frequency 


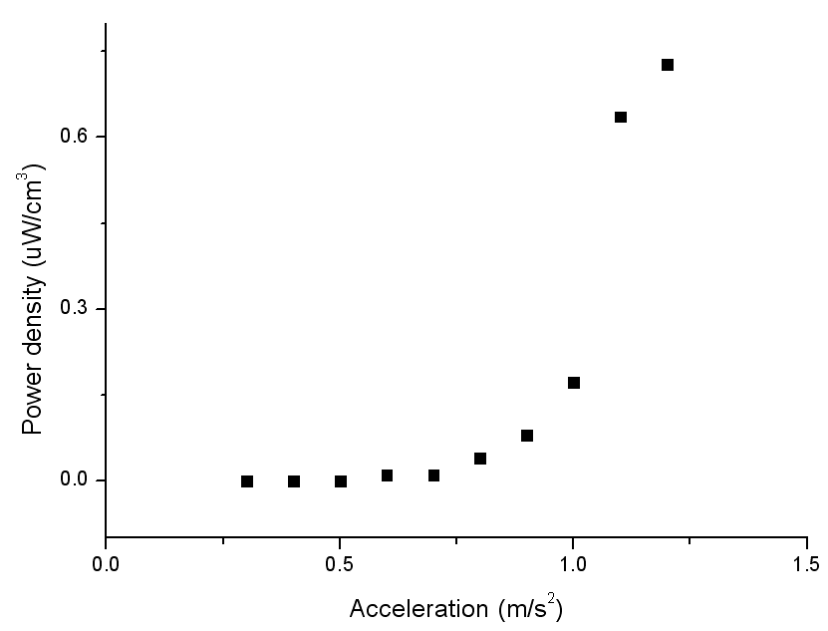

Fig. 6. Graph of power density versus vibration acceleration

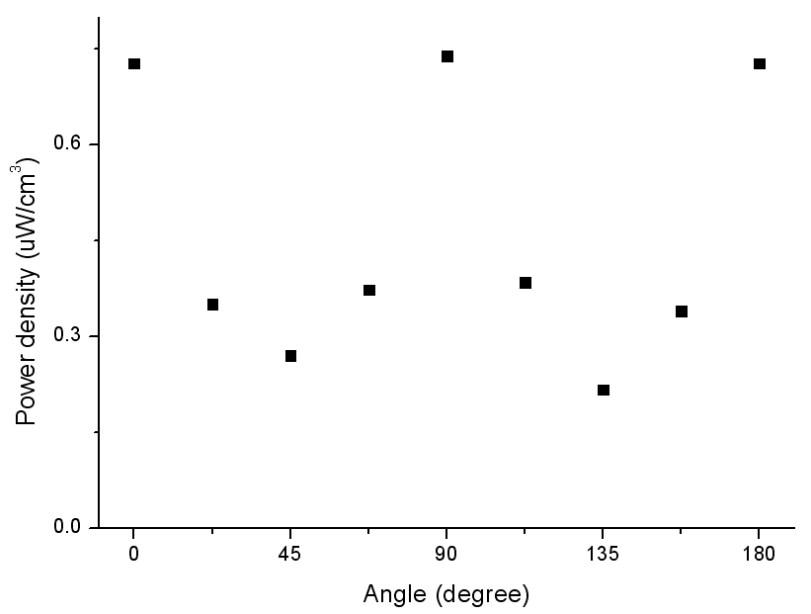

Fig. 7. Graph of power density versus clockwise angle of vibration direction from $\mathrm{x}$-axis

\section{Conclusion}

To respond variation of vibration direction, electrostatic energy harvester for two-axis vibration is proposed. Device size is $9.0 \mathrm{~mm} \times 9.0 \mathrm{~mm} \times 0.6 \mathrm{~mm}$ and silicon structure is anodic bonded on the glass substrate by semiconductor fabrication process. Unlike the energy harvester for one-axis vibration, the proposed device can generate energy when vibration direction changes from $0^{\circ}$ to $90^{\circ}$. The developed energy harvester can be used to machines which generates vibration of several directions. But the device has a limitation of vibration frequency. It should be improved to have wide bandwidth.

\section{References}

[1] S. Roundy, P. K. Wright, and J. Rabaey, "A study of low level vibrations as a power source for wireless sensor nodes", Computer Communications, Vol. 26, Issue 11, pp. 1131-1144, 2003

[2] S. Roundy, "Energy scavenging for wireless sensor nodes with a focus on vibration to electricity conversion", Ph.D. dissertation, Dept. Mech. Eng., Univ. California, Berkeley, CA, 2003

[3] P. Glynne-Jones, M. J. Tudor, S. P. Beeby, and N. M. White, "An electromagnetic, vibration-powered generator for intelligent sensor systems", Sensors and Actuators A, Vol. 110, Issues 1-3, pp. 344-349, 2004

[4] H. Külah, and K. Najafi, "Energy Scavenging From LowFrequency Vibrations by Using Frequency Up-Conversion for Wireless Sensor Applications", IEEE Sensors Journal, Vol. 8, No. 3, 2008

[5] N. M. White, P. Glynne-Jones, and S. P. Beeby, "A novel thick-film piezoelectric micro-generator", Smart Mater. Struct. Vol. 10, pp. 850-852, 2001

[6] S. Roundy, "On the Effectiveness of Vibration-based Energy Harvesting", Journal of Intelligent Material Systems and Structures, Vol. 16, pp. 809-823, 2005

[7] S. Roundy, P. K. Wright, and K. S. J. Pister, "MicroElectrostatic Vibration-To-Electricity Converters", Proceedings of IMECE'02, 2002 